\title{
General
}

\section{Thoracic Surgery}

\section{Outcome of bilateral lung volume reduction in patients with emphysema potentially eligible for lung transplantation}

Bryan F. Meyers, MD

Roger D. Yusen, MD

Tracey J. Guthrie, RN

Gail Davis, RN

Mary S. Pohl, RN

Stephen S. Lefrak, MD

G. Alexander Patterson, MD

Joel D. Cooper, MD
From the Division of Cardiothoracic Surgery and the Division of Pulmonary and Critical Care Medicine, Washington University School of Medicine, St Louis, Mo.

Read at the Eightieth Annual Meeting of The American Association for Thoracic Surgery, Toronto, Ontario, Canada, April 30-May 3, 2000.

Received for publication May 4, 2000; revisions requested Sept 5, 2000; revisions received Feb 12, 2001; accepted for publication Feb 19, 2001.

Address for reprints: Bryan F. Meyers, MD, Suite 3108 Queeny Tower, One BarnesJewish Hospital Plaza, St Louis, MO 63110 (E-mail: meyersb@msnotes.wustl.edu).

J Thorac Cardiovasc Surg 2001;122:10-7

Copyright (c) 2001 by The American Association for Thoracic Surgery

$0022-5223 / 2001 \$ 35.00+0 \quad \mathbf{1 2 / 6 / 1 1 5 2 3 9}$

doi: $10.1067 / \mathrm{mtc} .2001 .115239$
Objective: Between January 1993 and May 1998, we performed 200 consecutive bilateral lung volume reduction operations. After initial assessment, 99 of these patients were eligible for lung volume reduction and potentially eligible for immediate or eventual lung transplantation on the basis of age and absence of contraindications. All chose to proceed with lung volume reduction surgery. The outcomes of these 99 patients are reviewed to assess the consequences of proceeding with lung volume reduction surgery on patients potentially eligible for lung transplantation.

Methods: A retrospective study was performed with the use of a prospectively assembled computer database.

Results: The 61 men and 38 women were $55 \pm 7$ years old at evaluation for lung volume reduction. Mean values for first second expired volume, total lung capacity, and residual volume were $24 \% \pm 8 \%, 141 \% \pm 19 \%$, and $294 \% \pm 54 \%$ predicted. There were 4 operative deaths and 17 late deaths. Two-year and 5-year survival after evaluation for lung volume reduction are $92 \%$ and $75 \%$. The 32 patients who have been listed for transplantation after lung volume reduction include 15 who have undergone transplantation, 14 who remain on the list, and 3 who have been removed from the list. All 15 transplant recipients survived transplantation and 3 have subsequently died of rejection or late infection. The 12 living recipients have a median post-transplantation follow-up of 1.7 years. The age at transplantation was $58 \pm 5$ years with transplantation occurring $3.8 \pm 1.1$ years after lung volume reduction. Sixteen of 99 patients underwent lower lobe volume reduction with an increased rate of listing $(63 \%, P=.008)$ and transplantation $(38 \%, P=.003)$ compared with patients undergoing upper lobe volume reduction. Patients listed for transplantation were younger, more impaired, and experienced less benefit from lung volume reduction than patients not yet listed for transplantation.

Conclusions: The preliminary use of lung volume reduction in patients potentially suitable for transplantation does not appear to jeopardize the chances for subsequent successful transplantation. 


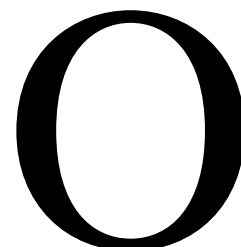

tto Brantigan initially proposed the concept of lung volume reduction surgery (LVRS) for emphysema by suggesting that resection of functionless, overinflated lung tissue might improve respiratory function. ${ }^{1,2}$ Several years ago, we reported our preliminary experience with bilateral LVRS. $^{3}$ Subsequent encouraging results from many centers have demonstrated significant improvements in pulmonary function tests and exercise tolerance along with reduction in oxygen use, reduction in subjective dyspnea, and improvement in subjective health status. ${ }^{4-6}$

We began performing LVRS in January 1993 and performed 225 bilateral operations over the next 6 years. The clinical results of this operation have been described. ${ }^{4}$ One of the initial driving forces to establish LVRS arose from the observation that many patients with severe emphysema who were referred for lung transplantation would never be eligible for transplantation. This ineligibility resulting from age or comorbidities identified a population of patients from which many of our earliest LVRS patients were drawn. As the efficacy and safety of LVRS became better established, it became apparent that LVRS might reasonably be applied to patients still under consideration for transplantation. Previously we offered lung transplantation only to patients with no other treatment option. However, the positive results after LVRS in patients ineligible for transplantation effectively created another surgical option for some transplant candidates: LVRS as a first step with potential for a lung transplantation at a later date. In some cases this LVRS may serve as a bridge to transplantation when a patient's life expectancy appears less that the anticipated waiting time for a lung transplant.

Our institutional bias has been that the best candidates for LVRS and the best candidates for lung transplantation constitute distinct groups with little overlap. ${ }^{7}$ However, because the natural history of emphysema with or without LVRS is one of progressive deterioration, we have frequently applied LVRS to individual patients with the expectation that many might later seek transplantation. It is these patients who make up the substance of this report. We sought to look retrospectively at the results of LVRS in a population of patients who were free of contraindications for transplantation at the time of the LVRS operation and who thus might become transplant candidates in the future.

\section{Methods \\ Patient Population}

We retrospectively searched our database to identify patients who had undergone bilateral LVRS who were, or could have been, considered candidates for transplantation at the time of LVRS. In general, patients with severe chronic obstructive pulmonary disease were selected for LVRS on the basis of the presence of a distended thorax and significant functional limitation despite standard medical therapy. Patients who underwent LVRS for giant emphysematous bullae, in the presence of nearly normal underlying compressed lung, were excluded from this report. Patients who continued to smoke were excluded from consideration. The details of the evaluation process have been fully described. ${ }^{8}$

The term transplant eligibility is an artificial classification that was retrospectively applied for the purposes of this report. To be considered transplant eligible, patients had to be less than 65 years of age and free of contraindications to lung transplantation. Figure 1 is a frequency histogram showing the age distributions of the 99 patients in this study, as well as the 267 patients with emphysema who have undergone lung transplantation in our program since 1988. The rarity of patients over the age of 65 years receiving transplantation in our program is justification of the use of this age as a cutoff for inclusion in this retrospective study. We placed no specific upper limit on lung function as part of this classification because the progressive nature of emphysema leads to eventual deterioration of pulmonary function in all of these patients. In keeping with our overall selection process for LVRS, the mean forced expiratory volume in 1 second $\left(\mathrm{FEV}_{1}\right)$ in this group of patients was $690 \pm 240 \mathrm{~mL}$, a value that is less than $25 \%$ predicted and represents substantial impairment.

\section{Assessment}

Physiologic assessment included standard pulmonary function studies, plethysmographic measurement of lung volumes, standardized 6-minute walk test, arterial blood gas values, quantitative nuclear lung perfusion scans, and a radionuclide cardiac ventriculogram.

Anatomic assessment included posteroanterior and lateral chest $\mathrm{x}$-ray films taken in inspiration and expiration, chest computed tomographic scan, and, in some patients, dynamic magnetic resonance imaging evaluation of chest wall and diaphragmatic movement and coordination. If pulmonary hypertension was suspected on the basis of the clinical examination or the right ventriculogram results, a right heart catheterization was performed. If significant coronary artery disease was suspected, catheterization of the left side of the heart was also done.

\section{Timing of the Operation}

After evaluation, virtually all patients were enrolled in a structured, supervised exercise rehabilitation program for a minimum of 6 weeks. In addition to the required 6-week rehabilitation delay between evaluation and surgery, additional delays were introduced on an individual patient basis to address issues raised at the time of evaluation. The interval between evaluation and LVRS in these patients ranged from 29 to 555 days with a median preparation time of 97 days.

\section{Technique of Operation}

The techniques used for the operation performed have previously been described. ${ }^{4} \mathrm{~A}$ thoracic epidural catheter was placed immediately before the operation, thereby eliminating the need for intraoperative systemic narcotic agents. A left-sided double-lumen endotracheal tube was used. The anesthetic management of these 


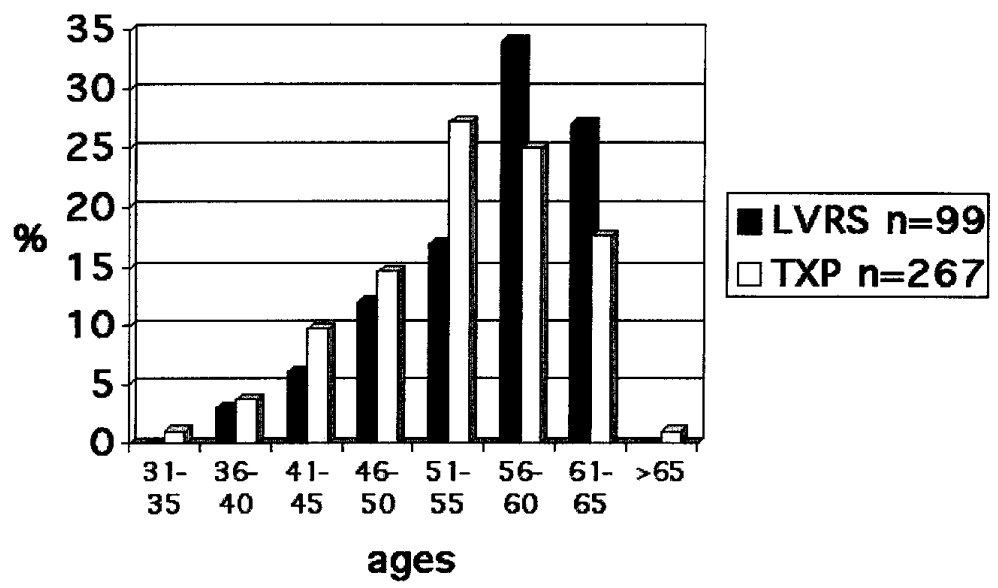

Figure 1. Frequency histogram showing age distribution of patients undergoing LVRS and lung transplantation (TXP). The LVRS group includes the 99 patients described in the current report. The lung transplantation group includes all patients with a diagnosis of emphysema who have undergone transplantation in the Washington University program since 1988.

patients has been previously described. ${ }^{9}$ A standard median sternotomy incision was made and the mediastinal pleura was incised several centimeters posterior to the sternum to facilitate subsequent closure of the pleura at the end of the procedure. Care was taken to avoid incising the pleura too far cephalad to avoid injury to the phrenic nerve. One lung was deflated while ventilation was directed to the contralateral side. Under these conditions, after a few minutes, the relatively healthy portions of lung undergo absorptive atelectasis whereas the most destroyed portions remain inflated because of the poor or absent pulmonary blood flow. In some instances, when the lobe in question was uniformly destroyed by emphysema, an anatomic lobectomy was performed in lieu of a stapled volume reduction.

A linear stapler was used to excise the selected diseased areas of lung. The staplers were buttressed with strips of bovine pericardium (Peri-Strips, Bio-Vascular, Inc, St Paul, Minn) using a technique previously reported. ${ }^{10}$ Two $28 \mathrm{~F}$ chest drains are placed in each pleural space and brought out in the subxiphoid position at the conclusion of the operation. Postoperative care has also been discussed in detail elsewhere. ${ }^{4}$

\section{Follow-up Evaluation}

Follow-up evaluation of all patients in both groups included telephone interviews and office visits to assess general health, functional status, and dyspnea. Overall patient satisfaction was queried by asking the following question: "On the basis of how you feel now, how would you rate the outcome of your volume reduction operation?" Patients were asked to choose from the following responses: 5 = excellent, $4=$ very good, $3=$ good, $2=$ fair, $1=$ poor. These interviews included questions regarding use of supplemental oxygen at rest and during exercise, as well as steroid use. We asked patients to return every 6 months for follow-up spirometry and lung volume measurements. Data describing survival, transplant listing, and actual transplantation are current as of March 2000 and functional data are complete as of November 1999.

\section{Statistical Analysis}

The mean and standard deviation were used to describe continuous data. Patient counts and percentages were used to describe categorical data. The 2-tailed Wilcoxon rank sum test was used to compare continuous data between groups. The Fisher exact test was used to compare categorical data between groups. Kaplan-Meier plots were used to graphically display survival, freedom from listing for transplantation, and freedom from transplantation, with the starting point for both groups being the day of evaluation for LVRS. The Mantel-Haenszel log rank statistic was used to determine the significance of survival differences.

\section{Results}

Between January 1993 and May 1998, we performed 200 consecutive bilateral LVRSs for patients with emphysema. Ninety-nine of these patients were considered potentially eligible for lung transplantation on the basis of age and absence of contraindications. The patients included 61 men and 38 women with an age of $55 \pm 7$ years. The $\mathrm{FEV}_{1}$ was $24 \% \pm 8 \%$ predicted, the residual volume was $294 \% \pm 54 \%$ predicted, the total lung capacity was $141 \% \pm 19 \%$ predicted, and the diffusion capacity for carbon monoxide was $34 \% \pm 17 \%$ predicted. The current status reflects a median follow-up of 5.1 years from evaluation with a range of follow-up from 2.2 to 7.1 years. The shortest follow-up from the actual LVRS is 1.9 years.

Ninety patients underwent the standard bilateral stapled volume reduction via a median sternotomy. In 74 patients the resection was for upper lobe predominant destruction, and in 16 the lower lobes were the site of resection. Nine of the 99 patients underwent a total of 11 anatomic lobectomies in the process of undergoing bilateral lung reduction. These operations included 6 right upper lobectomies, 1 left upper lobectomy, 2 right middle lobectomies, and 2 left lower lobectomies. 


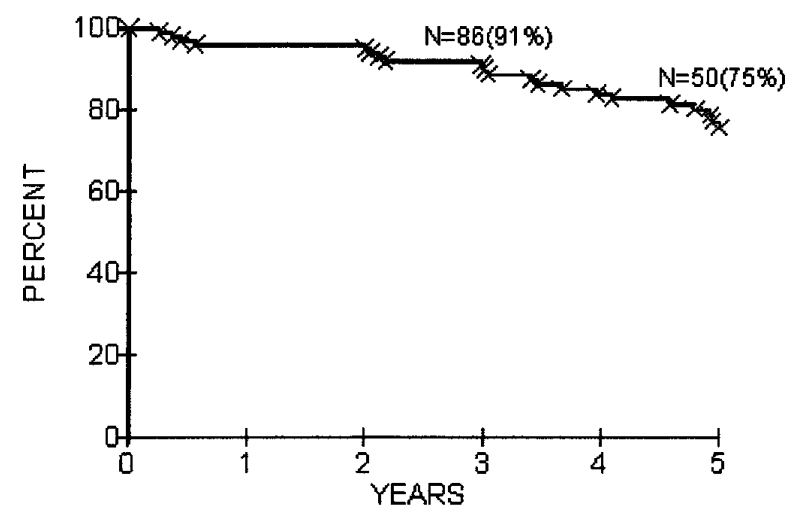

Figure 2. Kaplan-Meier curve showing survival of 99 potentially transplant-eligible patients after bilateral LVRS.

Four postoperative deaths occurred either in the initial hospitalization or within 30 days after LVRS. The overall survival of the 99 patients is depicted in Figure 2. The functional outcome is described by the changes in pulmonary function in the 80 patients with 6-month follow-up data. The $\mathrm{FEV}_{1}$ improved from $24 \% \pm 8 \%$ predicted at evaluation to $38 \% \pm 14 \%$ at 6 months' follow-up, an improvement of $56 \%$ from baseline. The residual volume decreased from $294 \% \pm$ $54 \%$ to $201 \% \pm 55 \%$ for a decrease of $32 \%$ of baseline. The 6- to 12-month overall patient satisfaction scores in the entire group show that most patients were quite satisfied with the outcome of surgery. There were 77 responses with a mean response of 4.4 and a median value of 5 on the previously described scale of 1 to 5 (poor to excellent).

To date, 32 patients have been listed for lung transplantation after receiving bilateral LVRS. Of the 32 listed patients, 15 have undergone transplantation, 14 remain on the waiting list, and 3 have been removed from the waiting list as a result of death or other reasons. Eight of the 14 patients are described as "active" on the waiting list, and 6 are considered "inactive" because they have accrued substantial time on the list but their lung function is considered to be too good to warrant transplantation at this time. Figure 3 is a Kaplan-Meier curve depicting freedom from transplant listing as a function of time since evaluation for LVRS. By 5 years from the time of LVRS, $40 \%$ of the survivors have been listed for transplantation. Table 1 compares the unlisted, listed, and transplanted patients according to selected demographic and baseline physiologic criteria. Listed patients are younger and have lower $\mathrm{FEV}_{1}$ scores at the time of evaluation than unlisted patients. Other physiologic parameters at the time of evaluation are not significantly different when the listed and unlisted cohorts are compared.

The 15 patients who have undergone transplantation ranged in age from 48 to 64 years at the time of transplan-
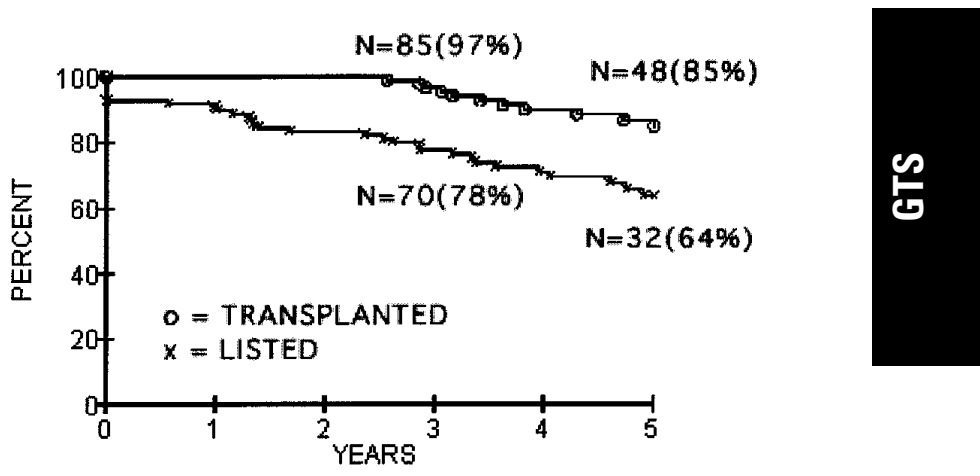

Figure 3. Kaplan-Meier curve showing freedom from transplantation and freedom from listing for transplantation of 99 potentially transplant-eligible patients after bilateral LVRS.

tation. The mean age was $57.5 \pm 5.1$ years. The mean time from LVRS to transplantation was $3.8 \pm 1.1$ years, with a range of 2.1 to 6.0 years. At the time of evaluation these patients were $53.9 \pm 4.6$ years with a range of 45 to 62 years of age. With the exception of the oldest patient in the group, no patient older than 58 years at the time of evaluation has undergone transplantation after LVRS. The initial improvement caused by LVRS in patients who subsequently went on to transplantation was comparable in many respects to the initial response experienced by patients not listed for transplantation. The increase in $\mathrm{FEV}_{1}$ at 6 months in these 15 patients was $47 \% \pm 35 \%$ over baseline, and the 6 - to 12 month patient satisfaction scores after LVRS were a mean of 3.8 and a median of 4 based on 13 responses. The 15 transplant recipients were re-evaluated just before transplantation, and the results were compared with physiologic indices obtained at the time of LVRS evaluation nearly 4 years previously. The fraction of these 15 patients requiring continuous use of supplemental oxygen at rest decreased from $62 \%$ to $23 \%$ between evaluation for LVRS and evaluation immediately before transplantation. The mean distance traveled in a 6-minute walk was 933 feet at LVRS evaluation and 1141 feet just before transplantation. The Medical Research Council dyspnea index improved from 3.2 at evaluation for LVRS to 2.9 before transplantation. The fraction of patients using supplemental oxygen at exercise increased from $92 \%$ to $100 \%$. All 15 patients survived transplantation. Three have subsequently died, and each death occurred more than 1 year after transplantation. The remaining 12 recipients are still alive with a median survival of 1.7 years (range $0.2-3.3$ years)

Figure 3 shows the overall Kaplan-Meier freedom from transplantation and Figure 4 shows freedom from listing for transplantation stratified according to 4 levels of preoperative $\mathrm{FEV}_{1}$. When patients are stratified by $\mathrm{FEV}_{1}$, the differences in transplantation risk are not statistically significant 
TABLE 1. Characteristics of patients listed for transplantation versus all patients not listed for transplantation

\begin{tabular}{lccc}
\hline Characteristic & Not listed for transplantation $(\mathbf{n}=\mathbf{6 7})$ & Listed for transplantation $\mathbf{( n = 3 2 )}$ & Pvalues, listed vs not listed \\
\hline Male/female & $50 / 17$ & $11 / 21$ & $<.001$ \\
Age at evaluation (y) & $56 \pm 7$ & $53 \pm 6$ & .01 \\
FEV $\%$ evaluation & $25 \pm 7$ & $22 \pm 6$ & .02 \\
RV\% at evaluation & $288 \pm 56$ & $307 \pm 49$ & .09 \\
TLC\% at evaluation & $141 \pm 20$ & $142 \pm 17$ & .99 \\
DLCO\% at evaluation & $36 \pm 17$ & $32 \pm 15$ & .10 \\
6 mo $\Delta$ FEV $\%$ & $65 \pm 45$ & $44 \pm 41$ & .04 \\
Lower lobe LVRS & 6 & 10 & .008 \\
$\quad$ Upper lobe LVRS & 61 & 22 & .13 \\
Patient satisfaction score at 6 mo & $4.6 \pm 0.7(\mathrm{n}=45)$ & $4.2 \pm 0.8(\mathrm{n}=17)$ &
\end{tabular}

$F E V$ \% , First second forced expiratory volume expressed as a percent of predicted; $R V \%$, residual volume expressed as a percent of predicted; $T L C \%$, total lung capacity expressed as a percent of predicted; DLCO\%, diffusion capacity for carbon monoxide expressed as a percent of predicted; $6 \mathrm{mo} \triangle \mathrm{FEV}, \%$, the improvement in the $\mathrm{FEV}_{1} \%$ expressed as a percentage of the value measured at evaluation. Patient satisfaction scores were recorded as whole numbers (1-5) with 5 being the best and 1 being the worst.

$(P=.18)$. Although only 1 transplant operation has been performed in a patient older than 60 years at evaluation, the difference in the transplant risks of the older and younger strata lacks statistical significance by the log rank test $(P=.09)$. The difference in the rate of progression to transplantation in the patients treated with lower lobe volume reduction as compared with upper lobe volume reduction is statistically significant by the log rank test $(P=.003)$, as shown in Figure 5 .

We have previously noted that the improvement in $\mathrm{FEV}_{1}$ after lower lobe LVRS is less than that seen after upper lobe LVRS despite the fact that the reduction in residual volume is similar. We have presumed that the physical properties of the remaining lung differ between these two types of emphysema, resulting in a less durable long-term result in patients with lower lobe predominant emphysema. The 16 patients receiving lower lobe bilateral LVRS were not significantly younger than patients having upper lobe LVRS ( $53 \pm 7$ years vs $55 \pm 6$ years; $P=0.27$ ), nor were they significantly more impaired at the time of evaluation $\left(\mathrm{FEV}_{1} 24 \% \pm 6 \%\right.$ vs $\left.24 \% \pm 7 \%\right)$. The lower lobe patients had a poorer objective response to bilateral LVRS, with an improvement in $\mathrm{FEV}_{1}$ measured at 6 months of $30 \% \pm 33 \%$ versus $63 \% \pm 45 \%$ for the upper lobe patients. As a consequence of this poorer result from bilateral LVRS, $10(63 \%)$ of the 16 lower lobe patients have been listed for transplantation as compared with 22 (27\%) of the 83 upper lobe patients, a difference that is statistically significant $(P=.008)$.

We investigated $\alpha_{1}$-antitrypsin deficiency as an independent risk for transplantation after LVRS. Ten of the 99 patients have been conclusively identified as having $\alpha_{1^{-}}$ antitrypsin deficiency. Because we did not routinely test patients with predominantly upper lobe disease for $\alpha_{1}$-antitrypsin deficiency, the overall prevalence of the condition within these 99 patients is not known. Six of the patients in whom this disorder was confirmed have undergone trans- plantation. Two of the remaining patients died without ever being listed (rectal abscess in 1 patient; Aspergillus, pulmonary embolus, and breast carcinoma in the other) and 2 patients are currently 64 and 65 years old and are not listed. Because patients with $\alpha_{1}$-antitrypsin deficiency frequently present with primary lower lobe destruction, and because of the poor results we observed after lower lobe LVRS, these patients are now preferentially referred for transplantation.

Twenty-three patients are currently alive, are unlisted for transplantation, and are considered too old to be listed. At the time of evaluation they were $61 \pm 2.0$ years old (range 57-64 years). These patients have now survived $4.3 \pm 1.2$ years after LVRS with a range of 1.4 to 6.3 years. At the time of evaluation, the $\mathrm{FEV}_{1}$ for this group was $24 \% \pm 5 \%$, the residual volume was $300 \% \pm 49 \%$, and the total lung capacity was $147 \% \pm 20 \%$. The initial response of this group of patients to LVRS as measured by 6 months' improvement in $\mathrm{FEV}_{1}$ was $66 \% \pm 28 \%$. The subjective patient satisfaction score in these patients 6 to 12 months after LVRS was a mean of 4.5 and a median of 5, with 18 of the 23 patients responding.

\section{Discussion}

The role of LVRS in patients who are eligible for transplantation continues to be defined. The decision is often difficult and requires a good deal of discussion with the patient and family. One particularly vexing issue is the relative value of an acceptable quality of life compared with length of life. For example, some evidence suggests that lung transplantation is a palliative operation that may actually shorten the life expectancy of the recipient with emphysema in exchange for relief of dyspnea and disability. ${ }^{11}$ This determination takes into consideration the stable survival trend observed in patients with chronic obstructive pulmonary disease on the lung transplant waiting list and the current 5year survival estimates of $40 \%$ to $50 \%$ after transplantation. 


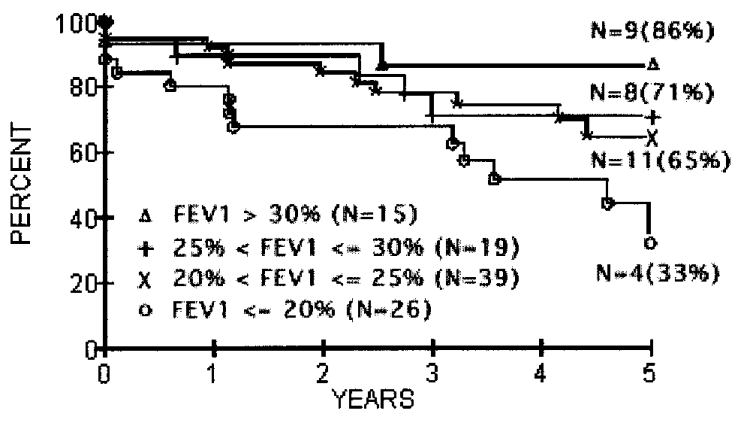

Figure 4. Kaplan-Meier curve showing freedom from listing for transplantation of 99 potentially transplant-eligible patients after bilateral LVRS stratified by FEV $_{1}$ at the time of evaluation.

On the other hand, most lung transplant recipients report a dramatic enhancement in the quality of life that may persist in a minority of patients for 10 years or more. By contrast, evidence to date suggests that both quality of life and longevity are improved with LVRS compared with nonsurgical treatment. We have previously published results in a group of Medicare patients who, having been selected for LVRS, were subsequently denied the operation because of the Medicare funding withdrawal in 1996. These patients were compared with an identical group of Medicare patients who received the operation in the year before the withdrawal of funding. ${ }^{12}$ At the time of our initial publication there was a trend toward improved survival in the surgical group, which has now reached statistical significance. ${ }^{13}$ Patients denied the operation had a median survival of 3.4 years whereas the median survival has not yet been reached at almost 5 years in the Medicare patients who received the operation. The National Emphysema Treatment Trial is currently attempting to prospectively address the impact of LVRS on survival in a multicenter trial.

Almost uniformly, patients with severe chronic obstructive pulmonary disease choose a surgical option (LVRS or transplantation) and its attendant risks over continued medical therapy, thereby assigning more importance to the quality of life than to longevity. Issues of quality and length of life notwithstanding, the surgical strategy for a "dual" LVRS and lung transplant candidate remains complex. The greater improvement in lung function offered by transplantation must be balanced against the lifelong need for immunosuppression and the continued risk for infection and chronic rejection. The waiting time for LVRS depends only on the length of preoperative rehabilitation, whereas the current waiting time for transplant recipients is 700 days in our program. If past trends hold, it is likely that patients listed today will routinely wait more than 2 years for suitable donor lungs. Finally, although no formal policy on age has been formulated, it is unlikely that a patient older than 65 years will be listed for transplantation.

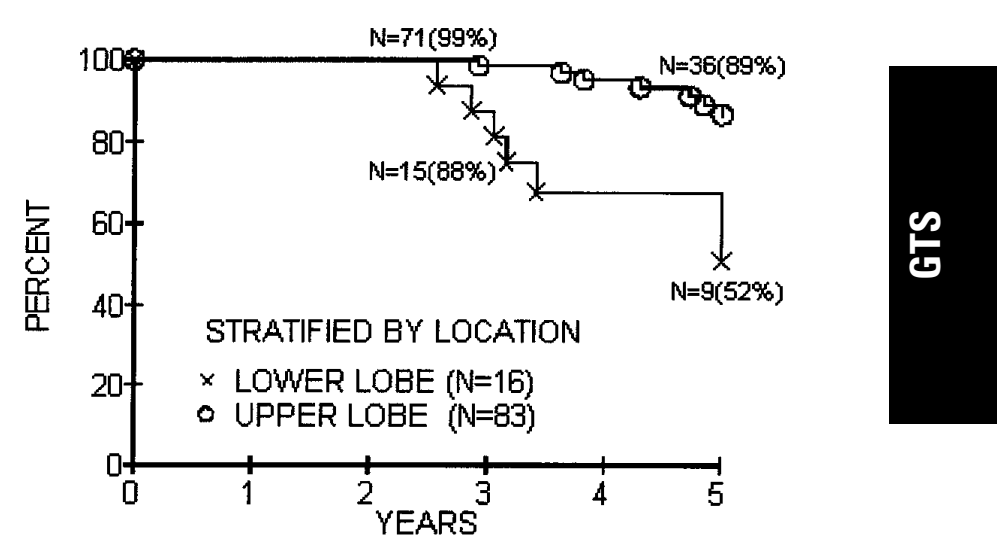

Figure 5. Kaplan-Meier curve showing freedom from transplantation in $\mathbf{9 9}$ patients undergoing bilateral LVRS stratified by site of LVRS: upper lobe versus lower lobe.

What can we advise a 55-year-old dual candidate about his or her likelihood of becoming a transplant recipient? What will be the impact of preliminary LVRS on the chances of obtaining a transplant and the success of a transplant if it is obtained? It is in this context that our results can provide guidance.

To analyze the impact of a particular surgical intervention, such as LVRS or transplantation, one can use the classic Kaplan-Meier method of describing freedom from "events" over a period of time from a starting point that is common to all subjects. Freedom from death, when measured as a function of time since evaluation or time since LVRS, is a reasonable measure of the effectiveness of the LVRS and of the judgment used to select surgical therapy for emphysema. Other events that can be used to chart the outcome for patients undergoing LVRS are the decision to list a patient for transplantation and the event of transplantation itself. With regard to listing and transplanting, both events can be viewed simultaneously as successes and failures of the initial therapy. The failure is clearly evident: an operation designed to improve symptoms of dyspnea has certainly lost its continuing benefit when the recipient of that operation undergoes lung transplantation. In young patients, however, this can be seen as a success. If the choices for a patient were previously limited to transplantation or medical therapy, and if the transplant had an 5\% to 8\% mortality and a $50 \% 5$-year survival, then a procedure such as LVRS that could palliate dyspnea, improve exercise capabilities, and delay entry onto the steeper post-transplantation survival curve would be most beneficial if it resulted in a transplant in every case. In that light, the critical measure would be time to failure as determined by time until transplantation or time until the patient's condition deteriorated to a functional and symptomatic level worse than that experienced before the procedure. 
Therefore, we must conclude that the goal of LVRS in these patients should be to provide relief from dyspnea, to produce an improved functional status, and to get the patient in optimal physical condition for subsequent transplantation. Eventual transplantation is expected, or even desired, but the role of LVRS is to palliate symptoms during the prolonged waiting period and to delay, as long as possible, the entry onto the steeper survival curve seen after lung transplantation. The question of what degree of palliation for what length of time is sufficient to merit the risks of LVRS is a value judgment. In our 15 patients who underwent transplantation, the time from LVRS to transplantation varied from 2.1 to 5.9 years. Although a longer latency from LVRS to transplantation is better, even the shortest palliation may be worthwhile if it enhances survival and quality of life and results in improved fitness and endurance at the time of actual transplantation.

Patients for whom successful LVRS may pose a dilemma comprise a subgroup worthy of additional scrutiny. An example would be a patient who, at the age of 61 years, would be considered a good candidate for either lung transplantation or LVRS. A successful LVRS would likely provide 3 to 5 years of palliation from the dyspnea that led to treatment in the first place, but when the progression of the emphysema reversed the results of surgery, such a patient would be viewed by many transplant programs as too old to list. Such a circumstance is likely to be rare, but the theoretical problem it represents is worthy of comment. One corrective measure would be to list the patient earlier than symptoms might otherwise dictate, but that runs into the moral dilemma caused by an overall shortage of donor lungs and the excessively high rate of death of sicker patients on the waiting list who are in more dire need of transplantation. At the time of this writing, almost half of the listed patients in this described experience who are alive and have not yet undergone transplantation $(6 / 14)$ are inactive on the waiting list. This status change has occurred because the patients were nearing the top of the waiting list in too good a condition to merit the risks of transplantation. This phenomenon suggests that we still have much to learn about the optimal timing of listing patients with emphysema for transplantation.

In conclusion, LVRS appears to be a reasonable strategy in highly selected patients eligible for LVRS and potentially eligible for transplantation at the time of evaluation. The outcome from LVRS in patients potentially eligible for transplantation appears comparable with that seen in all patients treated with LVRS. A minority of such patients will actually go on to transplantation, and the mean interval from LVRS to transplantation will be nearly 4 years. Patients undergoing transplantation are younger at the time of evaluation than patients not proceeding to transplantation. The initial improvement in lung function does not appear to discriminate between patients likely or unlikely to proceed to transplantation. Patients in whom lower lobe destruction is predominant appear more likely to require subsequent transplantation. In our experience with 15 patients to date, there has been no increased mortality in patients subjected to lung transplantation after prior LVRS.

\section{References}

1. Brantigan OC, Mueller E. Surgical treatment of pulmonary emphysema. Am Surg. 1957;23:789-804.

2. Brantigan OC, Mueller E, Kress MB. A surgical approach to pulmonary emphysema. Am Rev Respir Dis. 1959;80:194-202.

3. Cooper JD, Trulock EP, Triantafillou AN, Patterson GA, Pohl MS, Deloney PA, et al. Bilateral pneumectomy (volume reduction) for chronic obstructive pulmonary disease. J Thorac Cardiovasc Surg. 1995;109:106-16.

4. Cooper JD, Patterson GA, Sundaresan RS, Trulock EP, Yusen RD, Pohl MS, et al. Results of 150 consecutive bilateral lung volume reduction procedures in patients with severe emphysema. $J$ Thorac Cardiovasc Surg. 1996;112:1319-30.

5. Miller JI, Lee RB, Mansour KA. Lung volume reduction surgery: lessons learned. Ann Thorac Surg. 1996;61:1464-9.

6. Naunheim KS, Ferguson MK. The current status of lung volume reduction operations for emphysema. Ann Thorac Surg. 1996;62:60112

7. Meyers BF, Patterson GA. Lung transplantation versus lung volume reduction as surgical therapy for emphysema. World J Surg. 2001;25:238-43.

8. Yusen RD, Lefrak SS, The Washington University Emphysema Surgery Group. Evaluation of patients with emphysema for lung volume reduction surgery. Semin Thorac Cardiovasc Surg. 1996;8:83-93.

9. Triantafillou AN. Anesthetic management for bilateral volume reduction surgery. Semin Thorac Cardiovasc Surg. 1996;8:94-8.

10. Cooper JD. Technique to reduce air leaks after resection of emphysematous lung. Ann Thorac Surg. 1994;57:1038-9.

11. Hosenpud JD, Bennett LE, Keck BM, Edwards EB, Novick RJ. Effect of diagnosis on survival benefit of lung transplantation for end-stage lung disease. Lancet. 1998;351:24-7.

12. Meyers BF, Yusen RD, Lefrak SS, Patterson GA, Pohl MS, Richardson VJ, et al. Outcome of Medicare patients with emphysema selected for, but denied, a lung volume reduction operation. Ann Thorac Surg. 1998;66:331-6.

13. Meyers B, Yusen R, Lefrak S, Cooper J. Survival benefit seen after lung volume reduction surgery as compared to continued medical therapy for emphysema. Ann Thorac Surg. In press.

\section{Discussion}

Dr Douglas E. Wood (Seattle, Wash). I congratulate Dr Meyers and his colleagues at Washington University for yet another important analysis of their large experience in the surgical treatment of end-stage lung disease. The Washington University group have been consistent leaders in the development and evaluation of both LVRS and lung transplantation. They have also consistently obtained enviable results in both procedures and are in a position to show the best that can be expected in LVRS, lung transplantation, and in this case, LVRS in lung transplant candidates.

A quick overview of the results in this paper would seem to show that the glass is half empty or half full, depending on your perspective. Among 99 patients undergoing LVRS, there was a $4 \%$ early and $17 \%$ late mortality, with $32 \%$ of patients subsequently listed for lung transplantation. From one perspective, it would appear that LVRS failed in $53 \%$ of patients, because they either died or required transplantation. From yet another perspective, nearly $50 \%$ of patients who 
were candidates for lung transplantation have been able to delay or avoid transplantation. With the current shortage of organs, this may be a valuable outcome for these patients.

The goals of LVRS in transplant-eligible patients are 3-foldto avoid or delay transplantation or to improve quality of life while awaiting transplantation. The utility of LVRS will be dependent on the extent and durability of LVRS improvement; that is, how long of a delay is worth the morbidity and mortality of LVRS.

Dr Meyers, what is the algorithm that we should use for choosing LVRS or lung transplantation in patients who are candidates for both? You have chosen one subset of patients, those with lower lobe disease or $\alpha_{1}$-antitrypsin deficiency, as patients who should proceed straight to transplantation. What is the best procedure for the 45- to 55-year-old patient who wants to return to work? What is the best procedure for the 60-year-old patient who is extremely debilitated? Should we use $\mathrm{FEV}_{1}$ as a guide? You did not show significant differences in freedom from transplantation when choosing an $\mathrm{FEV}_{1}$ of less than $25 \%$, but there was a trend. What if one examined this with an $\mathrm{FEV}_{1}$ of less than $20 \%$ ?

Dr Meyers, you and your colleagues have consistently provided us with outstanding results, first in lung transplantation, and now with LVRS. The optimum management for these patients will depend on the summative morbidity and mortality of two sequential procedures, the differences in 5-year survival, and the extent and durability of quality of life changes after LVRS or transplantation. The National Emphysema Treatment Trial will provide the answers for LVRS, and the Transplant Registry data provide most of the information for transplantation, with a need for a more comprehensive examination of quality of life.

Dr Thomas M. Egan (Chapel Hill, NC). Congratulations on your results with a difficult operation in a difficult group of patients. We have not been quite so successful at the University of North Carolina, where we have done 3 transplants in patients who have had previous LVRS. The first 2 died, in part as a result of graft failure that was probably due to a prolonged ischemic time because of the difficulty encountered in performing pneumonectomy in these patients after LVRS. We have seen adhesions that are considerably worse than in the worst cases of cystic fibrosis. The third patient had a double lung transplant, and although his ischemic times were longer, I think the longer ischemic time was better tolerated by the double lung recipient than it was by the 2 single lung recipients who died.

How many of these patients had single lung versus double lung transplants, and did the decision to perform double lung transplantation relate to their previous LVRS and the anticipated difficulties that one would encounter doing pneumonectomies in this situation?

Dr Scott J. Swanson (Boston, Mass). I have another question along those lines. We have done some transplant operations after LVRS and have had issues with the phrenic nerve, although we have not seen any injuries. Do you have any tricks to avoid phrenic nerve injuries, and did you see any phrenic nerve injuries in this group?

Dr Stephen Yang (Baltimore, $M d$ ). Since you probably have the most experience, you are aware that there is a certain subpopulation of patients who require LVRS after transplantation. Can you speculate or do you have any data comparing that group versus the group that you just presented, regarding either exercise tolerance or pulmonary function?
Dr Meyers (St Louis, Mo). Thanks for all those comments.

Dr Wood, you mentioned failure, a word that we avoid when we discuss transplantation after LVRS. We view the consequent progression of the emphysema leading to transplantation as a predictable natural extension of the patient's pre-LVRS emphysema. Particularly with respect to the late transplantations or listings, they certainly should not be viewed as the consequence of failure of LVRS.

The harder part of your question was to set up an algorithm to offer optimal surgical therapy to these patients. Once one has made the leap and decided that a patient is actually truly eligible for both procedures, I think the dilemma is to involve the patient as much as possible in the decision-making process. The patients are the ones that will be exposed to the higher risk or the lower benefit, depending on how they decide. If you looked at unilateral LVRS, bilateral LVRS, and transplantation as surgical therapies with increasing benefit available to the patient, yet increasing risk to which they would be exposed, I believe each patient's personality would dictate how much morbidity and mortality should be risked for increased functional gain.

With regard to the age of the patient, of the multiple factors that we evaluated, several are true risks factors, whereas younger age is more of a permissive factor with regard to transplantation. We could not demonstrate a statistically significant difference with a cutoff at age 60 years, but I would say that only 1 patient who was 60 years or older at the time of LVRS subsequently went on to transplantation. That patient had the transplant operation at another center and likely would not have been offered transplantation at our center. Therefore, the algorithm is very difficult and by necessity involves careful consultation with the patient according to his or her comfort with the risks.

Dr Egan mentioned the difficulty of the pneumonectomies, and I would echo that. We have performed transplantation in a lot of patients who probably have chimeric lungs, with the cortex being the residual volume-reduced native lung and the medulla being the newly transplanted lung. In many cases we leave the visceral pleura and some lung tissue behind in the hopes of avoiding injury to the recurrent laryngeal nerve and the phrenic nerve.

I do not have information regarding the single and double lung question. I can tell you that 8 of the 15 transplants were performed by us at Washington University, and we definitely have a propensity toward bilateral transplantation. The other 7 were performed at other centers, and I would estimate that they were probably split evenly between bilateral and single lung transplantations.

Dr Swanson mentioned the hazards of dealing with phrenic nerves. Peri-Strips tend to have a magnetic attraction toward nerve tissue, particularly after 3 or 4 years of opportunity to migrate there. On many occasions we have just left the Peri-Strips and the attached lung stuck to the mediastinum in hopes of avoiding the nerves and the subsequent diaphragmatic injury. I cannot provide numbers, though, on the prevalence of phrenic or recurrent nerve injury resulting from these dissections.

Dr Yang inquired about LVRS after lung transplantation. To my recollection, we have only attempted that one time in a woman who had a very severe bronchiolitis obliterans after a single lung transplantation, and it proved not to be beneficial for her. The majority of our patients are receiving bilateral transplants, and so volume-reducing the native side is not a decision we are faced with very frequently. 